\title{
When the Mind Fails
}

A guide to dealing with incompetency 
This page intentionally left blank 


\section{When the Mind Fails}

A guide to dealing with incompetency

Michel Silberfeld - Arthur Fish

UNIVERSITY OF TORONTO PRESS

Toronto Buffalo London 
www.utppublishing.com

(C) University of Toronto Press Incorporated 1994

Toronto Buffalo London

Printed in Canada

ISBN 0-8020-0469-6 (cloth)

ISBN 0-8020-6780-8 (paper)

(

Printed on acid-free paper

\section{Canadian Cataloguing in Publication Data}

Silberfeld, Michel

When the mind fails : a guide to dealing with incompetency

Includes index.

ISBN $0-8020-0463-6$ (bound)

ISBN 0-8020-6780-8 (pbk.)

1. Mentally ill - Care. 2. Mentally ill aged Care. I. Fish, Arthur. II. Title.

HV3004.S55 $1994 \quad 362.2 \quad$ C93-094593-X 